\title{
Tuberculous Prostatic Abscess with Prostatorectal Fistula after Intravesical Bacillus Calmette-Guérin Immunotherapy
}

\author{
Jeong Ho Eom, Jai Hoon Yoon, Seok Won Lee, Hyo Sun Kim, Tae Young Park, Chang Seok Bang, Gwang Ho Baik and Dong Joon Kim \\ Department of Internal Medicine, Hallym University College of Medicine, Chuncheon, Korea
}

Intravesical bacillus Calmette-Guérin (BCG) immunotherapy is a common treatment modality for bladder cancer after transurethral resection of a bladder tumor. This therapy is generally safe, and development of a prostatic abscess with a prostatorectal fistula after intravesical BCG immunotherapy is a very rare complication. This finding was incidentally obtained by the authors, who examined a patient with colonoscopy for evaluation of abdominal pain. The patient was successfully treated with antitubercular drugs. To the authors' knowledge, this is the first report of a patient with a tuberculous prostatic abscess with prostatorectal fistula after BCG immunotherapy in South Korea. Clin Endosc 2016;49:488-491

Key Words: Prostate; Abscess; Fistula; Tuberculosis

\section{INTRODUCTION}

Bacillus Calmette-Guérin (BCG) has been commonly used as a vaccine against tuberculous infection. Intravesical BCG immunotherapy after transurethral resection for bladder tumor (TUR-BT) has been used to treat bladder cancer. ${ }^{1}$ This therapy is generally safe. It has a rare complication of tuberculous infection within the prostate. ${ }^{2}$ The complication of tuberculous prostatic abscess with prostatorectal fistula is extremely rare. Reported herein is a case of tuberculous prostatic abscess with prostatorectal fistula after intravesical BCG immunotherapy, which was successfully treated with antitubercular drugs.

Received: November 6, 2015 Revised: December 1, 2015

Accepted: December 1, 2015

Correspondence: Jai Hoon Yoon

Department of Internal Medicine, Hallym University Chuncheon Sacred Heart Hospital, Hallym University College of Medicine, 77 Sakju-ro, Chuncheon 24253, Korea

Tel: +82-33-240-5000, Fax: +82-33-241-8064, E-mail: yoonjh@hallym.or.kr

(cc) This is an Open Access article distributed under the terms of the Creative Commons Attribution Non-Commercial License (http://creativecommons.org/ licenses/by-nc/3.0) which permits unrestricted non-commercial use, distribution, and reproduction in any medium, provided the original work is properly cited.

\section{CASE REPORT}

A 61-year-old man presented himself to the gastroenterology department of the authors' hospital with a 1-month history of lower abdominal pain. Colonoscopy was performed to evaluate the abdominal pain, and a 6-mm rectal ulcer located on the anterior wall of the prostate, combined with a possible fistula tract, was observed (Fig. 1A). A biopsy of the rectal ulcer was taken for histopathological examination. The biopsy showed chronic granulomatous inflammation with caseation necrosis, consistent with tuberculosis (Fig. 2A). The biopsy specimen was positive for tuberculosis by polymerase chain reaction (TB-PCR).

The patient had undergone TUR-BT for a stage T1 bladder tumor 7 months ago. He had pulmonary tuberculosis when he was young, for which he had not had any treatment. A chest radiograph revealed signs of prior pulmonary tuberculosis. Intravesical BCG (12.5 mg in $50 \mathrm{~mL}$ normal saline, Oncotice; MSD, Kenilworth, NJ USA) immunotherapy was performed once a week for 6 weeks. There was no other significant medical history.

The rectal examination revealed an enlarged prostate, and no fistulous opening could be palpated. The microscopic urinalysis showed few leukocytes and few red blood cells. The 

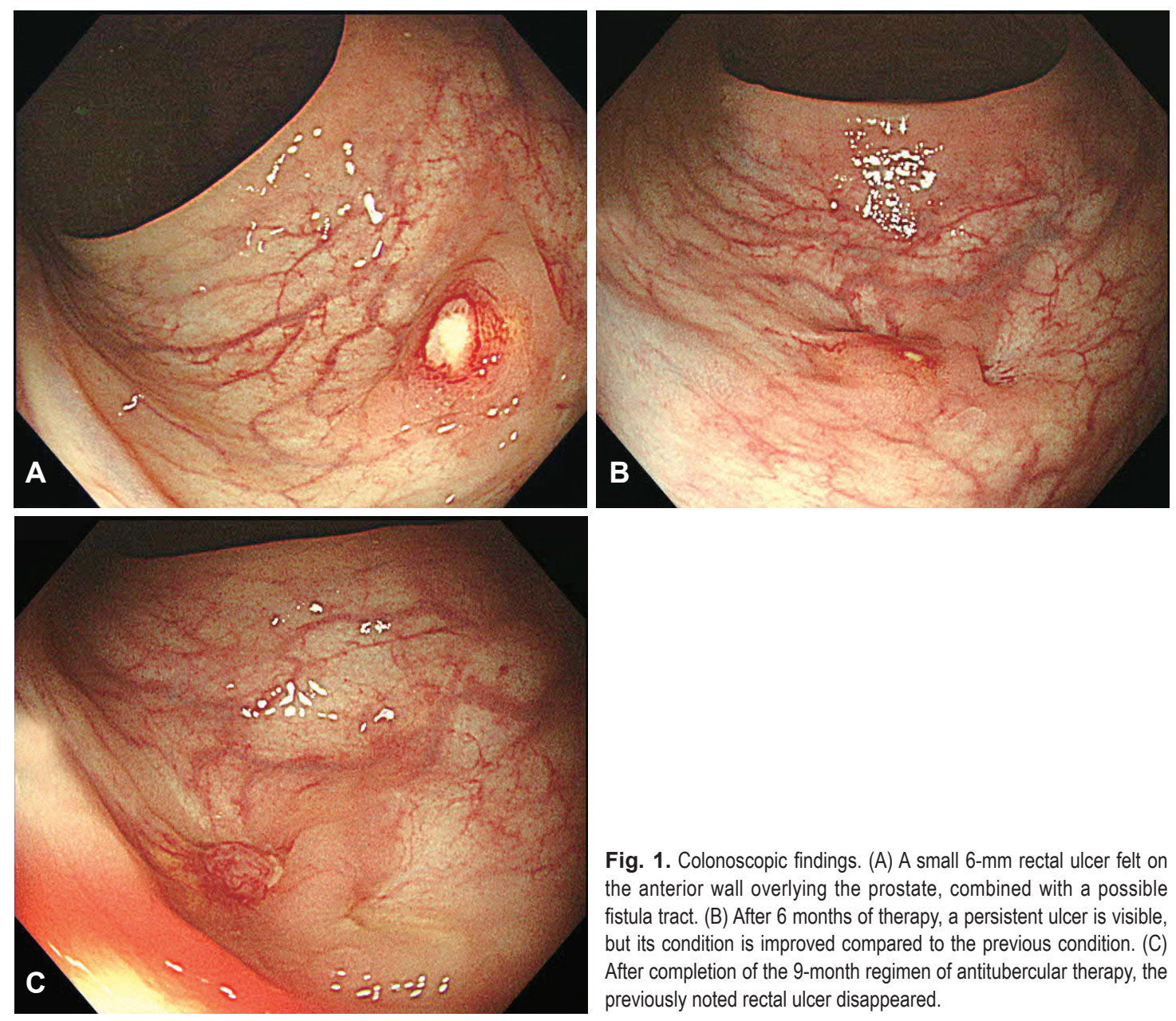

Fig. 1. Colonoscopic findings. (A) A small 6-mm rectal ulcer felt on the anterior wall overlying the prostate, combined with a possible fistula tract. (B) After 6 months of therapy, a persistent ulcer is visible, but its condition is improved compared to the previous condition. (C) After completion of the 9-month regimen of antitubercular therapy, the previously noted rectal ulcer disappeared.
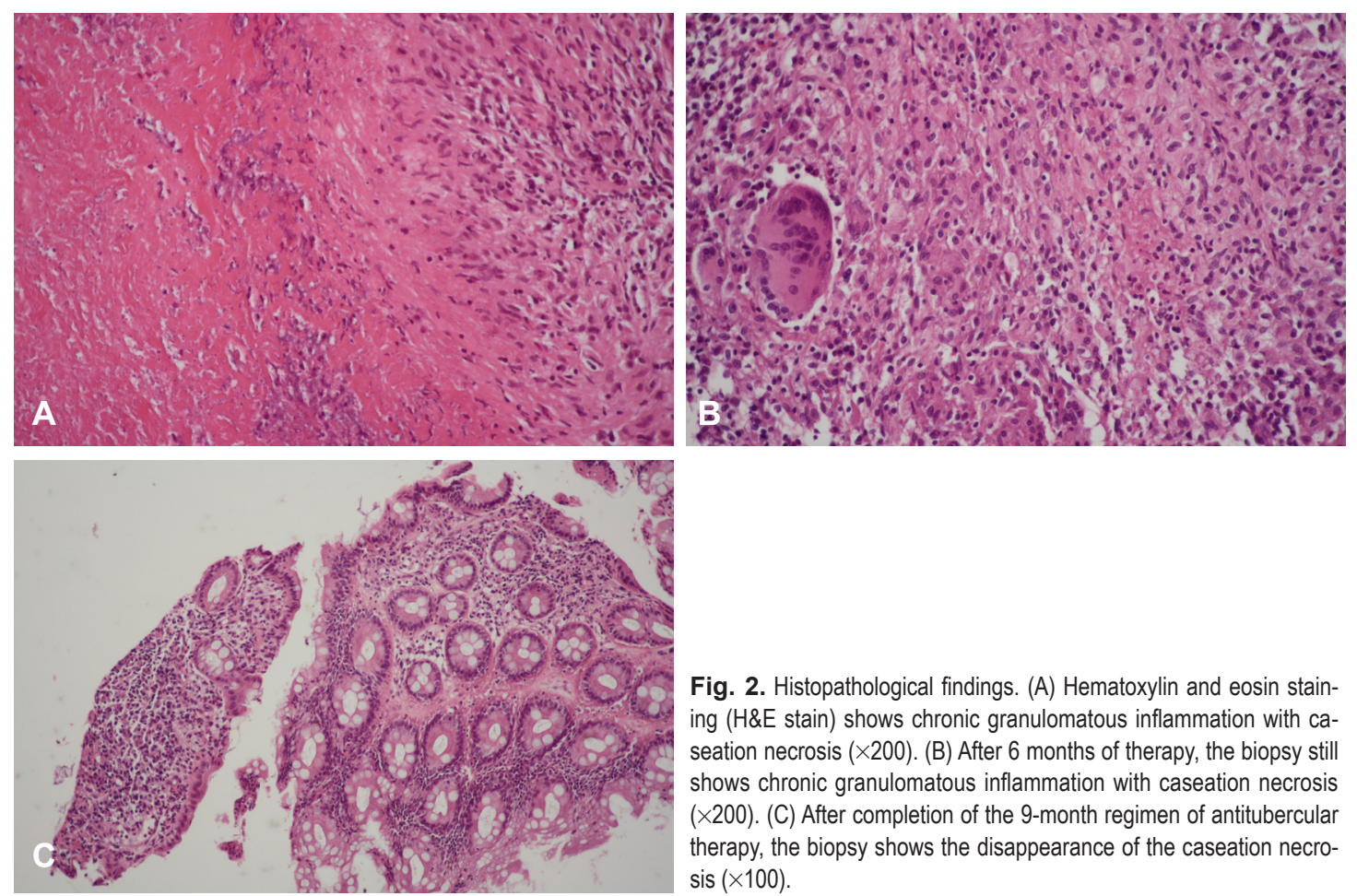

Fig. 2. Histopathological findings. (A) Hematoxylin and eosin staining (H\&E stain) shows chronic granulomatous inflammation with caseation necrosis $(\times 200)$. (B) After 6 months of therapy, the biopsy still shows chronic granulomatous inflammation with caseation necrosis (×200). (C) After completion of the 9-month regimen of antitubercular therapy, the biopsy shows the disappearance of the caseation necrosis $(\times 100)$. 

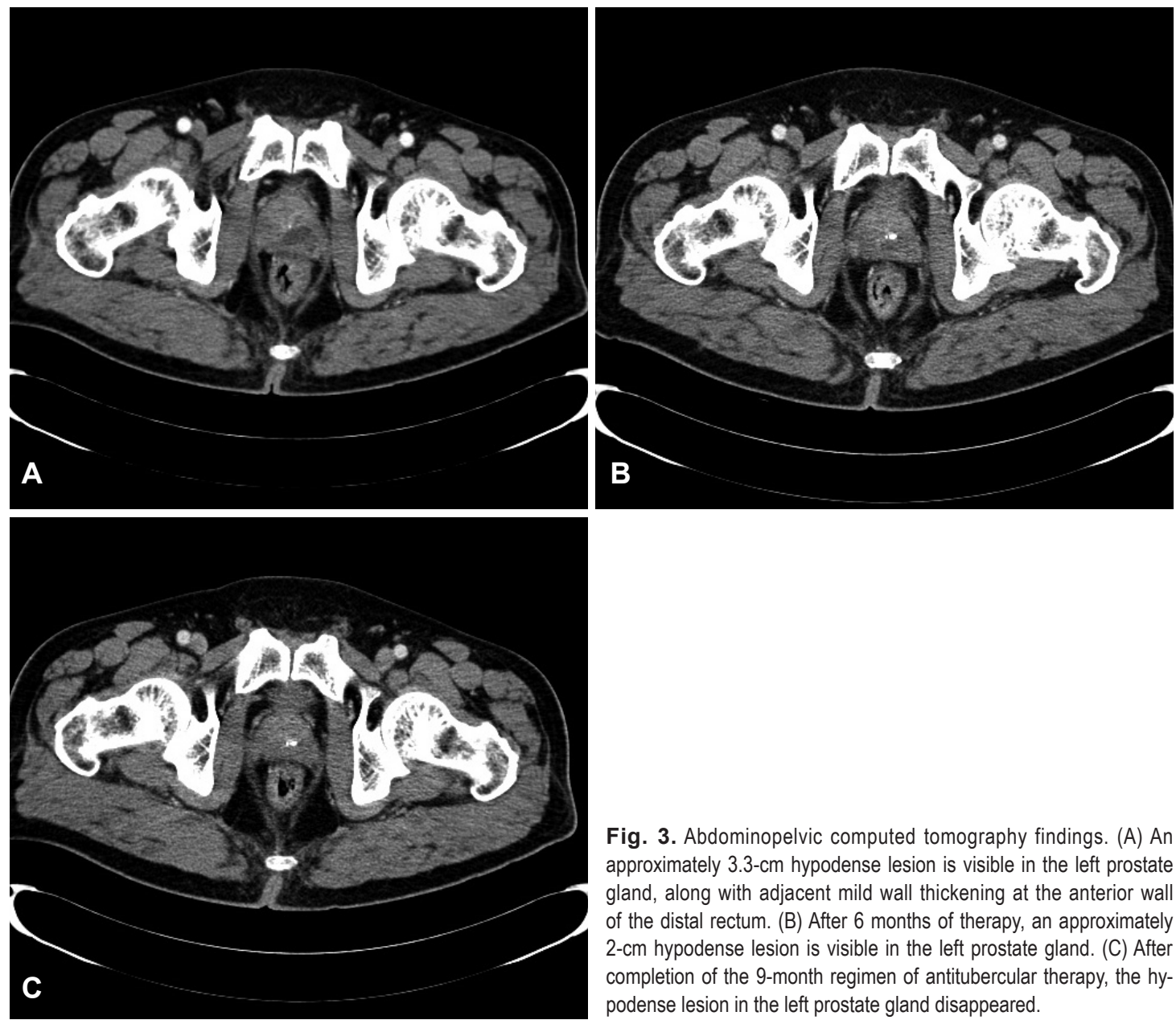

Fig. 3. Abdominopelvic computed tomography findings. (A) An approximately $3.3-\mathrm{cm}$ hypodense lesion is visible in the left prostate gland, along with adjacent mild wall thickening at the anterior wall of the distal rectum. (B) After 6 months of therapy, an approximately 2-cm hypodense lesion is visible in the left prostate gland. (C) After completion of the 9-month regimen of antitubercular therapy, the hypodense lesion in the left prostate gland disappeared.

urine was negative for acid-fast bacteria (AFB). The total prostate-specific antigen (PSA) was $2.7 \mathrm{ng} / \mathrm{mL}$, and the free PSA was $0.33 \mathrm{ng} / \mathrm{mL}$. An abdominopelvic computed tomography (CT) showed an approximately $3.3 \mathrm{~cm}$ hypodense lesion in the left prostate gland as well as adjacent mild wall thickening at the anterior wall of the distal rectum (Fig. 3A). Based on these findings, a diagnosis of prostatorectal fistula was made. The patient was treated with isoniazid ( $300 \mathrm{mg} /$ day), rifampicin (600 mg/day), ethambutol ( $800 \mathrm{mg} /$ day), and pyrazinamide (1 $500 \mathrm{mg}$ /day) for 2 months, followed by isoniazid $(300 \mathrm{mg} /$ day), rifampicin (600 mg/day), and ethambutol $(800 \mathrm{mg} /$ day $)$ for 7 months.

Transrectal ultrasonography was done a month after initiating the antitubercular therapy, and it showed a small hypoechoic lesion on the apical wall as well as a healed fistulous tract (Fig. 4). After 6 months of therapy, a follow-up colonoscopy was done, which showed a persistent ulcer but with an improved condition (Fig. 1B). A biopsy of the ulcer was taken. The biopsy specimen was negative for AFB but was positive for TB-PCR, and the biopsy still showed chronic granulomatous inflammation with caseation necrosis (Fig. 2B). The ab-

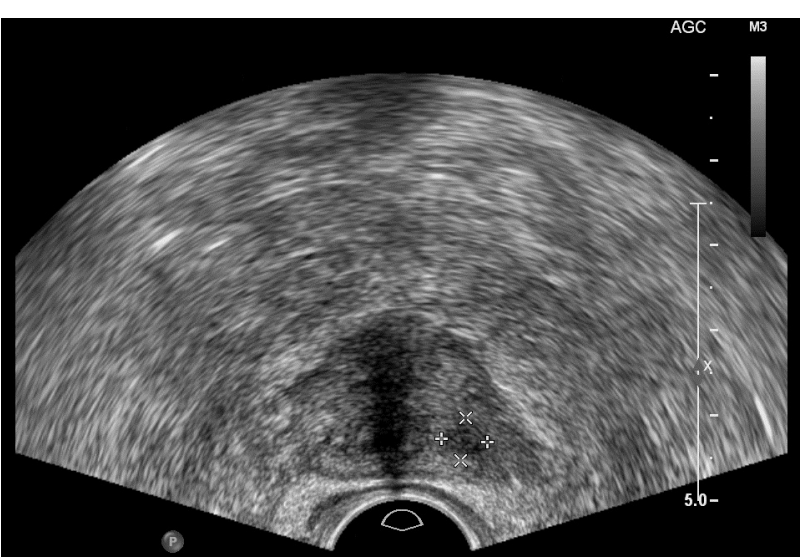

Fig. 4. Transrectal ultrasonography findings. The procedure, which was done a month after the antitubercular therapy was initiated, shows a small hypoechoic lesion on the apical wall as well as a healed fistulous tract.

dominopelvic CT showed an approximately 2 -cm hypodense lesion in the left prostate gland (Fig. 3B). The antitubercular therapy was continued for an additional 3 months. After completion of the 9-month regimen of antitubercular therapy, a follow-up colonoscopy was performed, which showed the dis- 
appearance of the rectal ulcer (Fig. 1C). The biopsy specimen from the site of the former ulcer was negative for TB-PCR, and the biopsy showed the disappearance of the caseation necrosis (Fig. 2C). The abdominopelvic CT also showed the disappearance of the hypodense lesion in the left prostate gland (Fig. 3C). The patient's latest cystoscopy showed no evidence of the prostatic abscess or fistula.

\section{DISCUSSION}

BCG has been commonly used as a vaccine for tuberculous infection. Furthermore, intravesical BCG immunotherapy after TUR-BT has been used for bladder cancer treatment. It was first used in 1976 as a non-specific immunostimulant. ${ }^{3}$ This therapy is generally safe, but it has a rare complication of tuberculous infection within the prostate, which can occur via hematogenous spread and through the direct extension of the infection. ${ }^{4}$ The direct extension of tuberculosis from the bladder may cause a prostatic abscess. Lamm et al. ${ }^{5}$ reviewed 1278 cases of bladder cancer treated with intravesical BCG immunotherapy and found that localized BCG infection was a rare complication. Its incidence rate is unknown, but tuberculous prostatic abscess with prostatorectal fistula is rarely reported and may be an extremely rare complication. This is because of the fascia located between the prostate and the rectum, which usually prevents fistula formation. ${ }^{6}$

Transurethral or transperineal drainage with 9 to 12 months of antitubercular treatment has been used as a tubercular prostatic abscess treatment modality. ${ }^{7.8}$ To the authors' knowledge, only five cases of prostate abscesses have been reported, of which three cases were treated with transurethral drainage, ${ }^{2,79}$ one with transperineal drainage, ${ }^{8}$ and one with only antitubercular drugs. ${ }^{10}$ Because the prostate abscess was small in the case presented herein, drainage was not performed, and the patient was treated only with antitubercular drugs. A follow-up abdominopelvic CT showed a completely healed prostatic abscess and fistula after 9 months of antitubercular treatment.

This is only the second report of a tuberculous prostatic abscess with prostatorectal fistula after BCG immunotherapy in the world, and the first report from South Korea. In the first-ever report of this complication, the patient was treated with transurethral drainage and antitubercular drugs. ${ }^{9}$ In the case presented herein, the patient was successfully treated with only antitubercular drugs. The results of this case indicate that conservative treatment with antitubercular drugs may be useful in treating tuberculous prostatic abscesses with prostatorectal fistulas. A larger-scale study will be needed for confirmation of this treatment's efficacy and safety.

In summary, tuberculous prostatic abscess with prostatorectal fistula is a very rare complication and the patient was successfully treated with antitubercular drugs only.

\section{Conflicts of Interest}

The authors have no financial conflicts of interest.

\section{REFERENCES}

1. Bellmunt J, Orsola A, Leow JJ, et al. Bladder cancer: ESMO Practice Guidelines for diagnosis, treatment and follow-up. Ann Oncol 2014;25 Suppl 3:iii40- iii48.

2. Kim SH, Kim HW, Lee HJ, Bae WJ, Cho SY. Tuberculous prostatic abscess following intravesical bacillus Calmette-Guerin instillation. Korean J Urol 2009;50:186-187.

3. Morales A, Eidinger D, Bruce AW. Intracavitary Bacillus Calmette-Guerin in the treatment of superficial bladder tumors. J Urol 1976;116:180183.

4. Lee HS, Lee SK. Tuberculosis in sex accessory organs. Korean J Urol 1999;40:259-262.

5. Lamm DL, van der Meijden PM, Morales A, et al. Incidence and treatment of complications of bacillus Calmette-Guerin intravesical therapy in superficial bladder cancer. J Urol 1992;147:596-600.

6. Kumar S, Kekre NS, Gopalakrishnan G. Diagnosis and conservative treatment of tubercular rectoprostatic fistula. Ann R Coll Surg Engl 2006;88:26

7. Aust TR, Massey JA. Tubercular prostatic abscess as a complication of intravesical bacillus Calmette-Guérin immunotherapy. Int J Urol 2005;12:920-921.

8. Matlaga BR, Veys JA, Thacker CC, Assimos DG. Prostate abscess following intravesical bacillus Calmette-Guerin treatment. J Urol 2002;167:251.

9. Enatsu N, Ota T, Ochi A. Prostatorectal fistula following intravesical bacillus Calmette-Guérin immunotherapy for carcinoma in situ of the urinary bladder. Int J Urol 2010;17:822-823.

10. Doo SW, Kim JH, Yang WJ, et al. A case of tuberculous prostatitis with abscess. World J Mens Health 2012;30:138-140. 